Review

\title{
Research Activities Arising From the University of Kent
}

\author{
Andrew C. LEWIN \\ Optical Sensors \& Systems Group, Technology Solutions Division, QinetiQ Ltd., UK \\ *Corresponding author: Andrew C. LEWIN_ E-mail: andyclewin@aol.com
}

\begin{abstract}
In this paper the author describes research and development activities in the field of optical sensing undertaken since his time within the Applied Optics Group at the University of Kent. The main topics covered are laser vibrometry and optical range-finding techniques. The author also gives a summary of his research at University of Kent, covering 1982-1985.
\end{abstract}

Keywords: Fiber, optic, sensor, vibrometer, interferometer, range-finder

\section{Activities at Kent}

My time in the Applied Optics Group at the University of Kent began after completing a first degree in Physics with Computing, also at the University of Kent. This whole period saw significant developments in both fiber technology and laser diodes, which jointly enabled the advancement of telecommunications architectures and also novel optical sensor systems.

My doctoral research concentrated on the use of fiber-based interferometric sensors, but also included a significant amount of investigation into and development of signal processing techniques relating to the interferometric sensor architectures. In addition to the core topics of my $\mathrm{PhD}$ work, the freedom that my $\mathrm{PhD}$ programme gave me also presented the opportunity to get involved to a lesser extent in other topics including fiber coupler manufacture, vortex-shedding flow-meters and laser diode characterization.

The key sensor types that I investigated were fiber gyros and fiber-based laser Doppler vibrometers. Although these two topics appear initially to be significantly different in concept, the signal processing techniques suitable for the demodulation of the phase information from each sensor are similar. Early work [1] had demonstrated that fiber sensors such as hydrophones could provide excellent sensitivity, but suffered from signal fading. The initial solution described involved locking the interferometer to a quadrature point (Fig. 1).

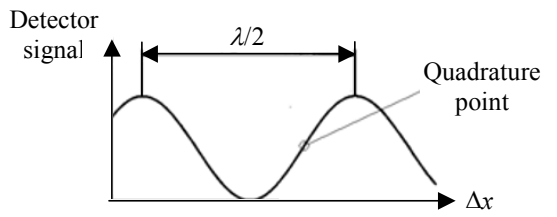

Fig. 1 Quadrature point of an interferometric signal.

This technique offered the ideal system sensitivity, but was not ideal in many cases due to the need for a servo control to maintain the operating point, and potential issues with larger signals where the phase excursion exceeded the linear region around the quadrature point. I developed a vibration measuring interferometer using a piezo-fiber-stretcher in the fiber section to enable the control of interferometric phase, as shown in Fig. 2 [2].

Received: 1 March 2011 / Revised version: 29 March 2011 


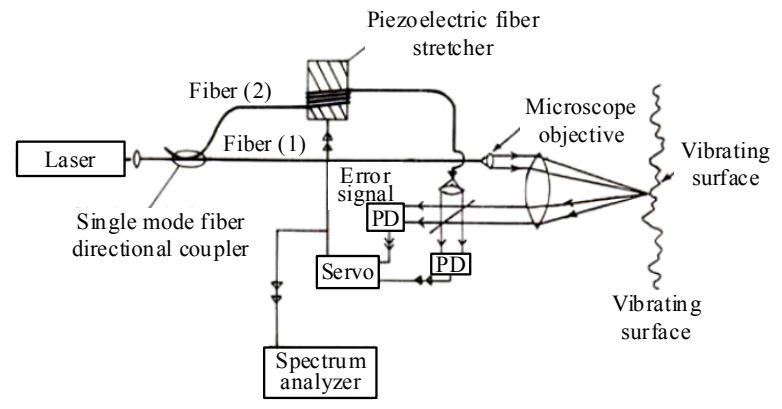

Fig. 2 A part fiber interferometer for evaluation of pseudo-heterodyne signal recovery techniques.

Earlier investigations on pseudo-heterodyne carrier generation [3] already showed that serrodyne modulation could be used to generate carrier signals that could be directly modulated by the measurand signal. As shown in Fig. 3 there are bandwidth limitations due to the sharp transients in the serrodyne drive signal.

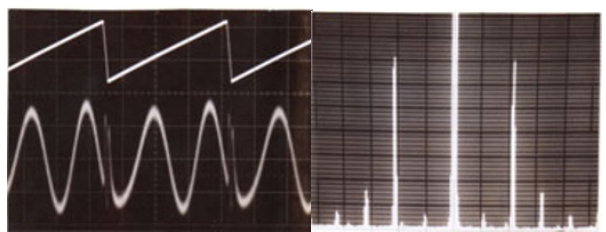

Fig. 3 Pseudo-heterodyne modulation of the interferometric phase to generate a carrier signal.

An alternative technique was developed which involved the use of sinusoidal modulation waveforms combined with additional postprocessing electronics [4] to recover the complete signal phase. This allowed the carrier frequency to be raised well above the anticipated signal frequencies, necessary for heterodyne type signal processing. It also allowed the piezo-electric modulator to operate at its resonant frequency to enhance system efficiency. Although there were various options available, the experiments used a $25 \%$ duty cycle square wave as the gating (multiplier) function, and a phase excursion of the modulator providing near to one complete cycle of interference fringe in the $25 \%$ section. The oscilloscope traces in Fig. 4 show the various signals generated, including the band pass filtered carrier.

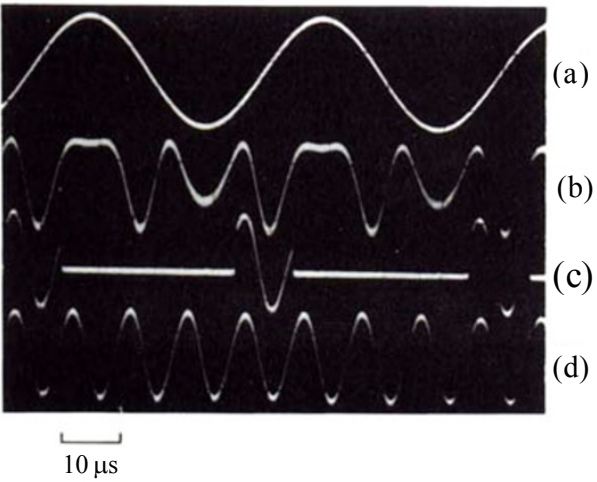

Fig. 4 A sinusoidal drive signal (a) generates a complex interference signal (b), which when suitably gated (c) and then band-pass filtered provides a carrier signal (d) which correctly represents the interferometric phase.

A key requirement is that the phase superimposed on the carrier signal should be directly (linearly) related to the interferometric phase. A mathematical model of the gating function combined with the phase modulation depth showed that specific selection of the settings was required, allowing the concept to be demonstrated.

A very similar signal processing technique was then also applied to the Sagnac interferometer [5] as shown in Fig. 5.

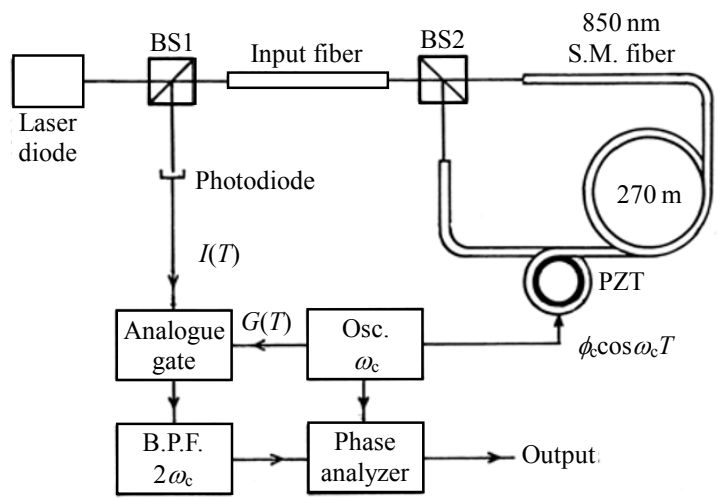

Fig. 5 A fiber gyroscope configuration which also uses pseudo/heterodyne carrier generation.

Unlike the vibrometer system, the Sagnac interferometer, which is the basis of the fiber gyroscope, has almost perfect zero path imbalance and is well suited to operation with a laser diode (as the phase noise will be small). Once again, the fiber stretcher is incorporated, placed at one end of the 
coil such that the two counter-propagating beams experience the phase shift at different times. This causes the output to show a phase shift dependant on the rate of change of drive signal, but once again, the sinusoidal drive technique allows this to be simply realized. System noise floors of the order 0.1 degree $/ \mathrm{sec}$ were achieved for the gyro, and $0.01 \mathrm{~nm} / \sqrt{ } \mathrm{Hz}$ for the vibrometer system.

The period when I was studying at the University of Kent also coincided with the arrival of the first laser diodes. I also carried out some additional work characterizing the new laser devices and investigating signal processing methods which utilized frequency modulation of laser diode sources. A further topic of investigation involved polarization based quadrature interferometers, both for vibrometry and gyroscopes, which allowed passive detection of the interferometeric phase [5] over a large dynamic range. These systems typically used quarter wave plates to arrange that the interference signals in two orthogonal polarization states were equivalent to a pair of quadrature signals. These quadrature signals, often denoted I and Q refering to in-phase and quadrature, equate to "sine" and "cosine" signals that could be used to calculate the interferometric phase.

Subsequent sections describe my research and development (R\&D) activities at Polytec $\mathrm{GmbH}$ (1986-2001) and QinetiQ, previously Defense Evaluation and Research Agency (DERA) (2001/2011).

\section{Activities at Polytec GmbH (1986-2001)}

\subsection{High stability fiber launchers}

The first interferometric sensors that I developed at Polytec were hybrid fiber optic/bulk optic systems [6] using Helium Neon (He-Ne) lasers and acousto-optic modulators (AOMs) to generate a true heterodyne signal. The fiber components of the interferometer allowed the alignment sensitive interferometric system to be decoupled from the measurement environment and associated targeting issues. In order to provide a fully developed product, one key aspect was the requirement for a robust fiber alignment system that could be set at manufacture and then shipped around the world to an engineer at the customer site such that the system would work "out of the box". The solution I developed [7] used a novel mechanical system featuring intertwined flexures allowing the rotation of a connector system in two axes about the center of the focussing lens, as shown in Fig. 6.

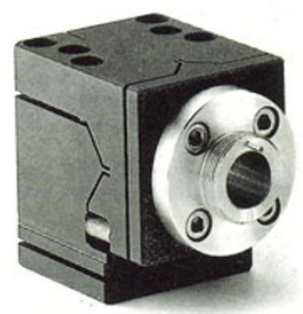

Fig. 6 Photo of stable fiber launcher (courtesy of Polytec $\mathrm{GmbH})$.

The third and fourth degrees of freedom were realized using flexures rotating about hinges at the extreme left and right of the base, again providing two orthogonal translational adjustments. These four axes were all designed to be accessible from above, and the final 2 degrees of freedom were realized within the connector itself via focus adjustment and a rotatable polarizing pin slot arrangement (Fig. 7).

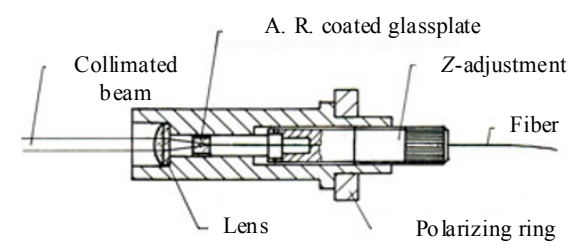

Fig. 7 A fiber launcher providing precise and robust adjustment of 4 degrees of freedom (courtesy of Polytec $\mathrm{GmbH})$.

Unlike fiber gyroscopes, strain sensors and other "intrinsic" fiber sensors, vibrometry applications involve the interference of a strong reference beam with a potentially extremely weak returning signal beam. It is therefore also critical that back/reflections into the interferometer are kept to 
an absolute minimum, so as to ensure that the required signal beam is not swamped with parasitic effects. These issues were addressed using two key methods. Firstly, the output connector incorporated a pre/aligned quarter wave plate (QWP) so as to return the signal beam to the interferometer in the orthogonal polarization state. Most of the back/reflections from the fiber ends were then de/selected by virtue of their polarization state. Polarization splitters are typically able to achieve extinction ratios of several hundreds to one, which is not suffcient on its own. In addition, anti-reflection (AR) coatings or angled polish with angled launching techniques were used to add further back-reflection reductions amounting to several orders of magnitude.

The launcher technology was a key enabling element in the design and development of single channel, dual channel and differential fiber vibrometers (Fig. 8) as well as fiber links for laser anemometry systems and other applications. The fiber vibrometers allowed flexible interferometric probes to be realized with typical diameters of $10 \mathrm{~mm}$ or $25 \mathrm{~mm}$. Special sensors were developed with even smaller dimensions for example to allow vibration and defect analysis between the plates of spinning computer hard drives [8].

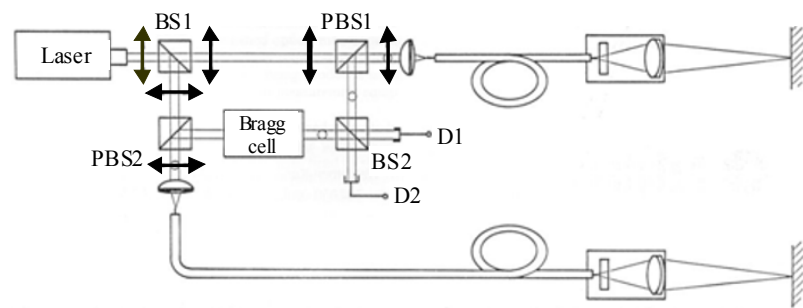

Fig. 8 The first polytec laser vibrometer was a differential 2 channel fiber based system using the launchers described above (courtesy of Polytec GmbH).

\subsection{Auto/aligned fiber interferometers}

Although the fiber interferometers offered the ultimate in sensor head miniaturization, these systems did always suffer some backscatter effects, which had an impact on system sensitivity. A new configuration was developed which used a "distant Fabry-Perot" as the effective interferometer [9]. The far end of the fiber was used as reference reflector, simply via the $4 \%$ Fresnel reflection at the glass/air interface. A bulk optic circulator was custom made for operation at $633 \mathrm{~nm}(\mathrm{He}-\mathrm{Ne})$, and a miniature Bragg-cell unit was developed to provide a very compact optical head. The unusual feature of the miniature Bragg cell was the bi-directional mode of operation, which allowed it to sit within the weak Fabry-Perot interferometer and generate a strong carrier at twice the running frequency. Figure 9 shows a block diagram of the architecture.

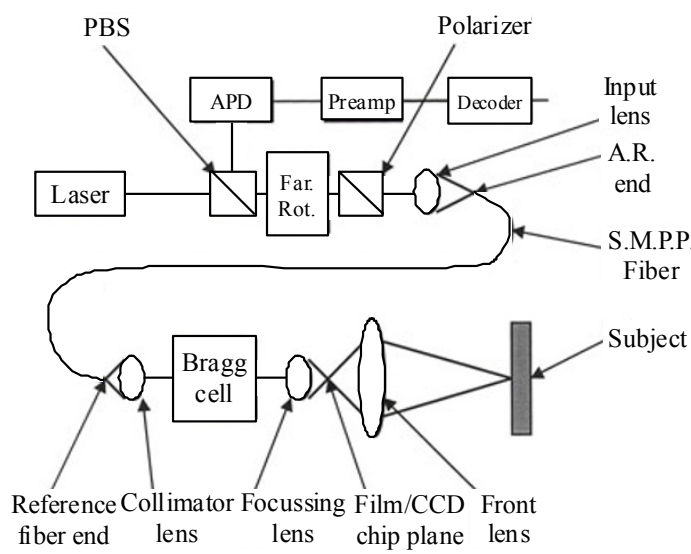

Fig. 9 Block diagram of the compact self-aligning interferometer using the distal fiber end as part of a remote Fabry-Perot interferometer (courtesy of Polytec $\mathrm{GmbH}$ ).

One key feature of this configuration is that the fiber is itself no longer part of the interferometer, which in fact starts at the fiber end, and reaches from there to the target of interest, where the beam is focussed. It should also be noted that, depending on the laser power available, the $4 \%$ fiber end reflection provides a reference (local oscillator) beam of marginal strength. The typical laser class 2 operation of such systems means that the reference power is only of order 40 microwatts, barely sufficient to boost the carrier signal above the detector noise of a normal pin photodiode. For this reason, an avalanche photodiode (APD) is typically used to maintain a suitably low noise floor. 
As mentioned above, one of the key aims of the development project is to generate a sensor head with very compact dimensions but with good system sensitivity. At this point it is worth defining two nominal parameters "sensitivity" and "resolution" in order to distinguish between two separate system limitations [10]. The diagram in Fig. 10 is used to demonstrate the key dependency.
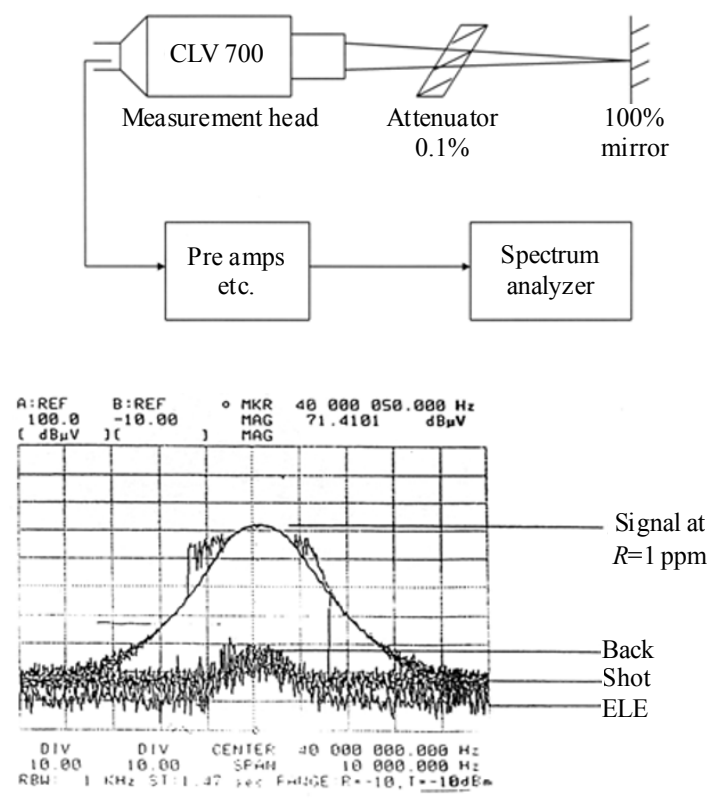

Fig. 10 Definition and measurement of system sensitivity and resolution: measurement setup above, results below (courtesy of Polytec GmbH).

In the heterodyne vibrometry configurations described here there is always a carrier signal generated, and this must be distinguishable above the noise floor in the chosen bandwidth for a signal to be suitably detectable. Longer integration (averaging) times result in smaller bandwidths and thus better (lower) noise floors, however if we fix the measurement bandwidth, useful comparisons can be made. In order to define a point of reference, the probe beam is focussed on a (near) $100 \%$ mirror via a $0.1 \%$ transmitting attenuator (ND3), as shown in Fig. 10. This generates a return signal equivalent to a 1 part per million (ppm) reflectivity. Note that the attenuator must be clean and pure (no scatter) and tilted to ensure that back-reflections do not swamp the well controlled $1 \mathrm{ppm}$ reflection. A plot of the radio frequency (RF) spectrum at and around the carrier frequency can then be taken to determine some of the system characteristics. Firstly, the height of the carrier above the broader band (white) noise is a key parameter which we can define as "resolution". Vibration signals (or more generically, sinusoidal interferometric phase changes) generate frequency modulation (FM) sidebands whose amplitude is given by:

$$
A_{\mathrm{s}}=\mathrm{J}_{1}(\varnothing / 2) \approx \varnothing / 2 \text { (for small signals) }
$$

where $\varnothing$ is the peak phase deviation.

For the case of the pure bulk-optic interferometer represented in the lower plot of Fig. 10, the carrier is seen to be about $50 \mathrm{~dB}$ above the white noise floor. This value can be used to determine how small a vibration amplitude can be "resolved", i.e. seen above the noise in this resolution bandwidth.

On the lower plot of Fig. 10 there are three further traces marked, the first of which is "Back" referring to the background carrier level. This is the carrier signal which results from either back reflections and scatter within the system optics, or possibly a residual signal at the carrier frequency resulting from electrical crosstalk from the Bragg cell drive. It is this "background carrier" level that determines the "sensitivity", i.e. optical return signal level at which the sensor can no longer demodulate the signal of interest, but sees an apparent target with velocity zero. In practice, the system performance degrades well before this level due to corruption of the vibration signal. The separation between the chosen reference signal and the level denoted "Back" (here $\sim 44 \mathrm{~dB}$ ) is thus a measure of the systems "sensitivity".

Two further traces are also noted in Fig. 10, and the first of which is "Shot", the shot noise floor. In an ideal system, this is the fundamental noise floor that should be the dominant noise source. As the return signal is several orders of magnitude below the local oscillator (LO or reference) beam power, 
the LO will be the dominant contributor towards shot noise. By blocking the outgoing probe beam within the interferometer (just after the split), the detectors are arranged to only see LO power, and the trace captured represents the level of broad band noise. If the system has been designed correctly, this level should be purely derived from shot noise in the LO, which can be tested by blocking the laser output. The final trace (ELE) represents the noise contributed by the electronic system, typically from the detector pre-amplifiers. Provided this level is a further 3 or more $\mathrm{dB}$ below the $\mathrm{LO}$ derived noise floor, the system is deemed to be "shot noise limited".

Figure 11 shows a comparison between the auto-aligned fiber vibrometer (CLV) and a near-ideal bulk optic system (Polytec OFV-300). As can be seen, the background carrier for the CLV can be almost $10 \mathrm{~dB}$ higher than the bulk optic system, but varies from plot to plot. This is due to multiple reflections, which interfere with one another found to originate in the two lenses which make up the front telescope/beam expander. Note also that, even though the reference beam is relatively weak, the APD ensures that the detector noise is well below the shot noise level.

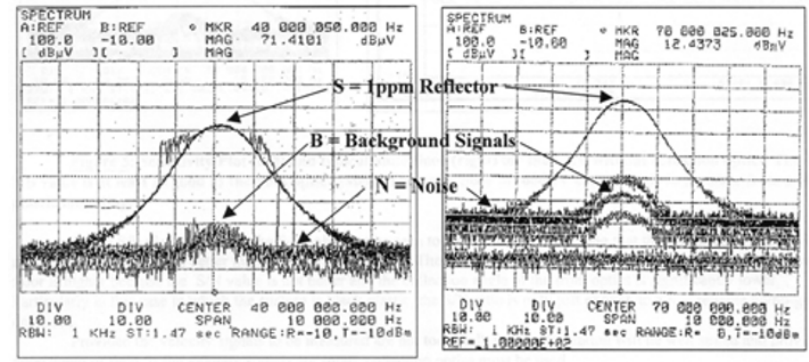

Fig. 11 CLV system performance (right) compared to a full bulk optic system (left)(courtesy of Polytec $\mathrm{GmbH}$ ).

The resultant system is then much smaller than a bulk-optic interferometer but with almost the same sensitivity and resolution, a far superior performance than the original fiber interferometer systems. The compact and robust (self-aligning) nature of the system has allowed this unit to be used in both harsh environments (automotive testing) and delicate environments such as diagnostics of the human ear [9].

\subsection{Multi-point \& 3D systems}

The pure bulk-optic, hybrid self-aligning and original fiber-optic vibrometers also formed the basis for a range of extended capabilities. Scanning systems were developed to allow sequential multi-point vibrometry which allowed the generation of full vibrational mode analysis of objects from fly's ears [11] up to complete cars. The standard bulk-optic system was coupled to galvo driven mirrors to allow wide area scanning with high sensitivity, as shown in Fig. 12.

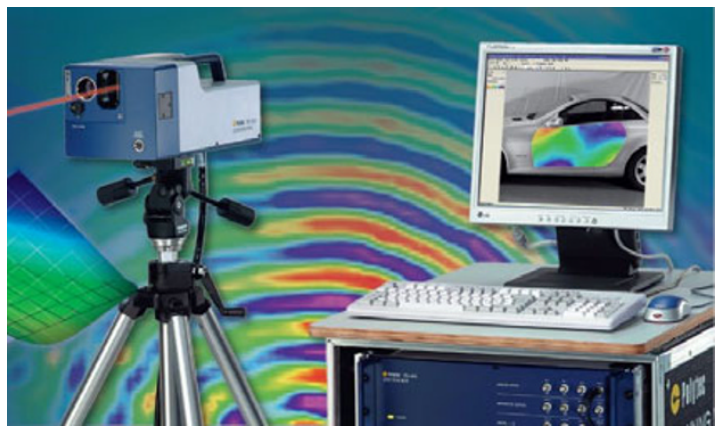

Fig. 12 Scanning vibrometer systems for the visualisation of vibration characteristics of medium to large (cars) objects (courtesy of Polytec GmbH).

The compact size and good sensitivity of the CLV systems were put to good use to arrange 3 probe beams through a single large front lens, allowing the detection of three velocity components and thus the $3 \mathrm{D}$ vibration characteristics of a target.

Adaptations of the various vibrometer types were also constructed, in response to customer and market demands, to allow analysis of components and objects at the other end of the size scale. The left hand image in Fig. 13 shows the vibrational mode of a frequency selective micro-electro mechanical systems (MEMS) accelerometer from an airbag release system. Each microscopic finger is designed to oscillate at a chosen resonance frequency, the operation of which could be verified using the 
system. In the case of the fly, studies were performed to help understand how this particular fly (Ormia ochracea) appears to have good directional hearing even though the spacing between its ears is so small $(0.5 \mathrm{~mm})$ compared to the wavelength in question. Studies using the scanning vibrometer showed that there is an unusual mechanical coupling between the two tympanal membranes, which enables the fly to detect the direction of its prey even though this would not initially appear possible.

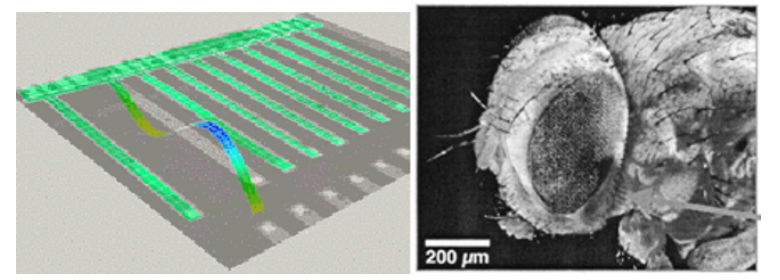

Fig. 13 Compact and microscope scanning versions allowed the investigation of MEMS components and fly's ears (courtesy of Polytec GmbH).

\subsection{Pseudo-heterodyne sensors}

Many years after leaving Kent, a strange specialized requirement led me back to the original pseudo-heterodyne interferometer architecture. An extremely compact but sensitive system was required for inclusion within a ultrasonic wire bonding machine [12]. During the intermediate years, laser diode technology had advanced sufficiently that usable coherence length sources were now commonly available. By using the fiber end reflection as reference mirror, as in the $\mathrm{He}-\mathrm{Ne}$ based CLV system, but replacing the "intra-etalon" Bragg cell with a laser diode modulation generated pseudo-heterodyne effect, a very small sensor head could be realized.

Due to the limited frequency modulation abilities of the "typical" laser diode, an etalon length must be selected which is long enough to allow modulation of the interferometer phase over a few cycles of the interference pattern without driving the laser diode injection current over too large a range. Intensity modulation is the primary effect generated, which means that an amplitude modulated (AM) signal is always present and must be corrected out if the modulating signal has a significant amplitude. Conversely, the phase noise within the interferometer is kept to a minimum if the optical path length is kept short. A compromise must then be selected which is long enough to allow modulation to be achieved successfully, but short enough to keep the noise level low. A trade off study resulted in an etalon length of $76 \mathrm{~mm}$. The sensor head is shown attached to a wire bonder head in Fig. 14.

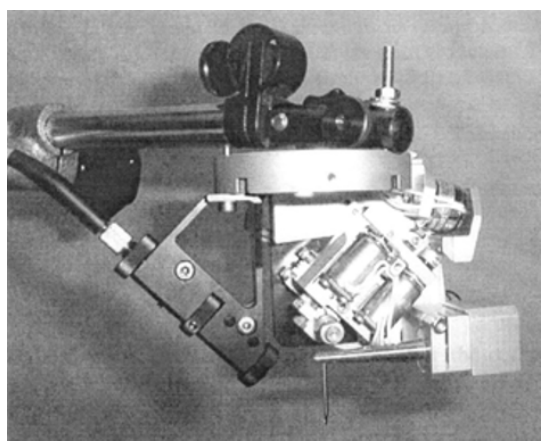

Fig. 14 Sensor attached to a wire bonder head (courtesy of Polytec $\mathrm{GmbH})$.

After installation of the sensor in a functioning bonder, it was found that the loaded vibration characteristics of the bonder could successfully be measured and used to help replicate the correct amplitudes at the different phases of the bonding process.

\subsection{Other work at Polytec}

The sections above describe the main areas of my R\&D work at Polytec in the period from 1986 to 2001. Additional topics of interest include laser diode based laser Doppler flowmeters, rotational vibrometers [13], surface velocimeters (see Fig. 15 below) and in-plane vibrometers. These four product types all utilize Doppler difference configurations to determine velocity components orthogonal to the main optical axis of the system. Probably the most challenging (and eventually rewarding) development task I undertook in this area was the surface 
velocimeter for use in the measurement of the line speed of hot steel in rolling mills.

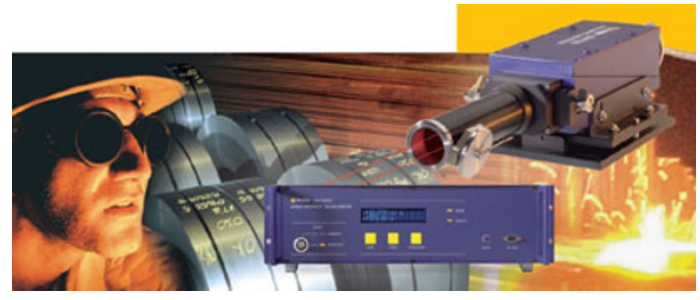

Fig. 15 Laser surface velocimetry operating in the extremely challenging hot steel mill environment (courtesy of Polytec $\mathrm{GmbH})$.

This technology provides key information for the control loops that regulate the pressure applied to the rollers. The key feature is that, with a given material feed rate, the speed of the material exiting the rollers is indicative of the thickness present directly between the rollers, even if the speed measurement is made some distance downstream of the compression point.

\section{Activities at QinetiQ UK}

\subsection{Multi-point range-finding}

In 2001, I moved to QinetiQ UK (formerly DERA) to join the Lasers and Photonics Group. After a few smaller projects related to adaptive optics systems [14], I was tasked with analyzing and developing methods for optical 3D shape measurement. The initial intention was to utilize wavefront sensing to determine the range to spots projected onto the object of interest, but during the analysis it became clear that a higher spot count could be achieved using multi-channel triangulation. A key challenge that had resulted from commercial discussions was an aversion to the term "laser", so one of the primary goals was to be able to generate a large number of low divergence probe beams without the use of a laser source.

A novel light emitter diode (LED) based transmitter unit [15] was developed which used a simple kaleidoscope principle to collect most of the light from an LED source and convert it into thousands of well defined low divergence probe beams. Figure 16 shows the basic layout in which a 5-element LED array attached to one end of a square glass rod provides numerous apparent sources alongside the one real one to generate a grid of low divergence beams.

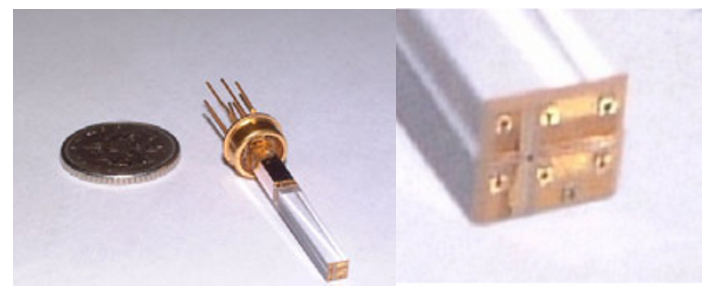

Fig. 16 Efficient non-laser based probe beam generation using an LED array attached to a kaleidoscope (multiple reflections along the waveguide rod generate many apparent sources).

The fact that each virtual source appears to project a narrow cone of rays towards the exit aperture of the kaleidoscope rod ensures that the individual beams have low divergence, and that all of the light from the LEDs is used efficiently.

A source of this nature was then placed next to a low-cost camera module and arranged to detect the parallax effect from each of the beams, effectively providing multiple triangulation based range-finder systems in a simple assembly [16]. A second novel feature was the specific configuration and the calibration routines to complete the sensor. The apparent position of any given spot will clearly move across the charge coupled device (CCD) sensor as a function of range, as for any single point rangefinder of this type. The tracks corresponding to each spot travel along a line parallel to that which links the projector and camera axes. A large number of spots are required for good shape measurement, so ambiguities are a key issue.

By ensuring that the square grid of points is rotated slightly $(\sim 1$ in 7$)$ relative to the lines, the spot tracks lie alongside one another for some distance before collision occurs, ensuring minimal ambiguities [17]. The image and shape plot shown in Fig. 17 demonstrate the effect with a limited 
number of points. The final product shown in Fig. 18 contained six 3D camera units each producing 4800 measurement points.

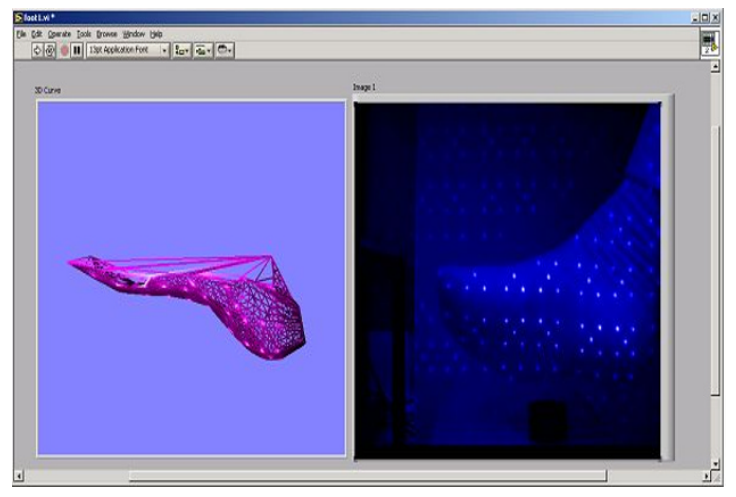

Fig. 17 Projection of the spot pattern onto a foot and the resultant multi-point range data representing shape.

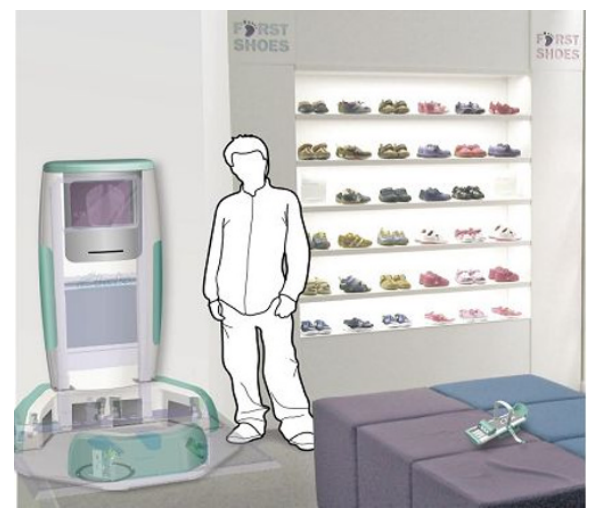

Fig. 18 Final shape measurement system as developed for Clarks provides six sets of 4800 range values (courtesy of Clarks).

Not only does this capability provide reliable non-contact shape measurement data for shoe fitting, but the full shape information can be used to optimize future shoe designs to provide a better fit.

\subsection{Precision range-finding}

A particularly interesting range-finding challenge that has led to one of the latest programmes I have undertaken is the precision position measurement of formation flying satellites. Many future satellite missions require large effective apertures which will be achieved using sparse phased array configurations. The European Space Agency (ESA) has therefore instigated a series of technology missions to evaluate the feasibility and reliability of such architectures, and QinetiQ was one of the companies funded to develop a candidate system. Our approach [18] makes use of a hybrid technology containing features from interferometry and amplitude modulated continuous wave (AMCW) range-finding in order to provide the long range capability of AMCW but also interferometric attributes. Figure 19 shows the fundamental mode of operation.

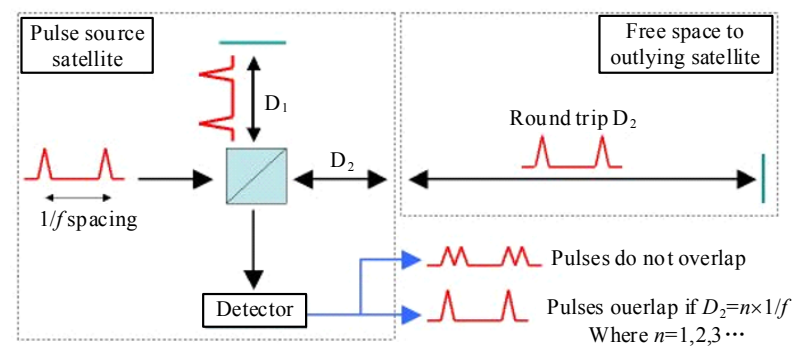

Fig. 19 Mode of operation of the scanning interferometric pulse overlap detection (SIPOD) system.

A telecommunications laser and modulator are used to generate a pulse stream whose frequency is derived from a precision clock. This pulse stream is amplified using an erbium doped fiber amplifier (EDFA) and injected into a small core fiber in which non-linear compression sharpens the pulses, if required, to near picosecond width. This pulse stream is injected into an interferometer where one arm is retained within the first satellite and the second is directed towards a corner cube on the second satellite. A specialized "quadrature upshifter" circuit is used to fine tune the reference oscillator over a small range, thus scanning the repetition rate of the optical pulse source. As shown in Fig. 19, interference effects can only occur when the two pulse streams are recombined with coincident arrival of the returning pulses. A set of two or more precise repetition rates can thus be found which correspond to the conditions under which maximum interference occurs, corresponding to frequencies where an integer number of pulse separations fit within the optical path length difference of the 
interferometer. Once two or more repetition rates have been found which satisfy the integer fit requirement, and absolute range can be determined and the system can switch to an interferometric tracking (fringe counting) mode. A fiber interferometer has been constructed to provide a miniature, light-weight optical head design for the range-finder, as shown in Fig. 20.

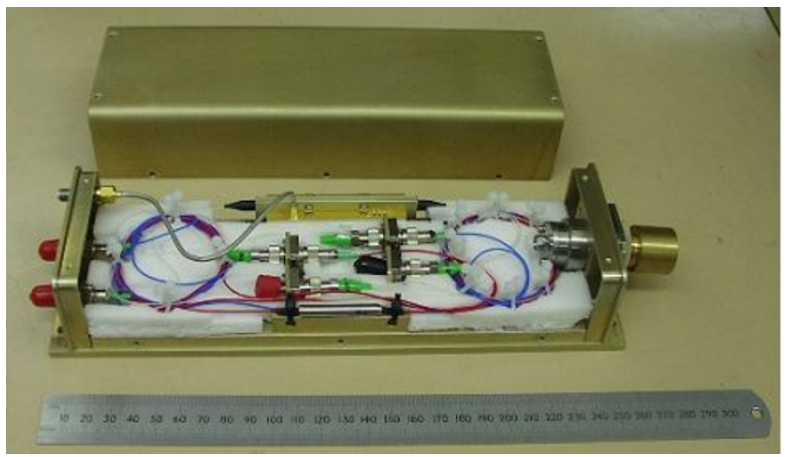

Fig. 20 SIPODs fiber Mach-Zehnder interferometer incorporating balanced phase modulators for carrier generation and symmetric circulators for output coupling.

The prototype system uses a $6 \mathrm{GHz}$ master oscillator (later potentially $\mathrm{Rb}$ reference source) and has been shown to be able to determine absolute range out to $2 \mathrm{~km}$, and at the nominal operating range of $250 \mathrm{~m}$, to provide 1.7 micron precision. A key issue is now that of finding a terrestrial system capable of verifying the theoretical accuracy. Further optimization of the pulse compression system could be used to further reduce the system noise, which could potentially bring the accuracy within the interferometric range. At this point, the technology could provide "absolute" interferometry as opposed to a relative measurement, as the interferometric order could be determined.

\subsection{Other work at QinetiQ}

Alongside the topics described above, I have also led several projects on security and defence related systems, which are not reported here. Other topics of interest include ongoing vibrometry work and also retro-based communications systems utilizing MEMS technology.

\section{Acknowledgement}

The work reported here warrants the acknowledgement of a great many colleagues and collaborators from the University of Kent, Polytec $\mathrm{GmbH}$ and QinetiQ limited. I am indebted to Professor David Jackson for the initial opportunity to work at Kent as a PhD student back in 1982, and also Julian Jones, Alan Kersey and others for their support and encouragement. At Polytec, the enthusiastic drive from Helmut Selbach and strong collaboration from Kai Wichdorff, Georg Siegmund and others were also invaluable. Finally at QinetiQ, numerous colleagues have also been instrumental in evolving new ideas, technologies and products, which have become vital for the ongoing success of the group.

Open Access This article is distributed under the terms of the Creative Commons Attribution License which permits any use, distribution, and reproduction in any medium, provided the original author(s) and source are credited.

\section{References}

[1] D. A. Jackson, R. Priest, A. Dandridge, and A. B. Tveten, "Elimination of drift in a single mode optical fiber interferometer using a piezo-electrically stretched coiled fiber," Applied Optics, vol. 19, no. 17, pp. 2926-2929, 1980 .

[2] D. A. Jackson, A. C. Lewin, A. D. Kersey, M. Corke, and J. D. C. Jones, "Open air path/fiber interferometer for remote vibration analysis," presented at European Conference on Sensors and their Applications, UMIST, Manchester, September, 1983.

[3] D. A. Jackson, A. D. Kersey, M. Corke, and J. D. C. Jones, "Pseudo-heterodyne detection scheme for optical interferometers," Electronics Letters, vol. 18 , no. 25 , pp. 1081-1083, 1982.

[4] A. C. Lewin, A. D. Kersey, and D. A. Jackson, "Non-contact surface vibration analysis using a monomode fiber interferometer incorporating an open air path," Journal of Physics E, vol. 18, no. 7, pp. 604-608, 1985.

[5] A. C. Lewin, A. D. Kersey, and D. A. Jackson, "Two wide-dynamic-range signal-recovery schemes for the 
fiber-optic gyroscope," in IEE Proceedings (opto-electronics), vol. 132, no. 5, pp. 271-276, 1985.

[6] H. Selbach and A. C. Lewin, "Differentielles fiberoptisches laser Doppler vibrometer zur Schwingungsanalyse," presented at Laser 87 Optoelectronics in Engineering, Munich, Germany, October, 1987.

[7] A. C. Lewin and K. V. Wichdorff, "Fiber links for optical instrumentation," Laser und Optoelektronik, vol. 20, no. 4, pp. 54, 1988.

[8] A. C. Lewin, F. Mohr, and H. Selbach, "Heterodyne Interferometer for Vibration analysis," Technisches Messen-tm, no. 9, pp. 335-345, 1990.

[9] A. C. Lewin, "Compact laser vibrometer for industrial and medical applications", SPIE (3rd International Conference on Vibration Measurement by Laser Techniques: advances and applications), vol. 3411, pp. 61-67, 1998.

[10] A. C. Lewin and G. Siegmund, "The implications of system 'sensitivity' and 'resolution' on an ultrasonic detecting LDV," SPIE (1st International Conference on Vibration Measurement by Laser Techniques: Advances and Applications), vol. 2358, pp. 292-304 1994.

[11] D. Robert and A. C. Lewin, "Scanning laser vibrometry applied to the biomechanical study of a small auditory system," SPIE (3rd International Conference on Vibration Measurement by Laser Techniques: Advances and Applications), vol. 3411, pp. 564-571, 1998.

[12] A. C. Lewin and F. Ritter, "Laser diode based pseudo-heterodyne interferometer for on-line process control of a wire bonder," SPIE, vol. 4072, pp. 8-18, 2000.

[13] A. C. Lewin, V. Roth, and G. Siegmund, "New concept for interferometric measurement of rotational vibrations," SPIE (1st International Conference on Vibration Measurement by Laser Techniques: Advances and Applications), vol. 2358, pp. 24-36, 1994.

[14] S. C. Woods, A. C. Lewin, et al., "Measuring wavefront properties," Patent WO 03074984, September 12, 003.

[15] A. C. Lewin and D. A. Orchard, "Structured light projector," Patent WO 2004044523 , May 5, 2004.

[16] A. C. Lewin, D. A. Orchard, and S. C. Woods, "Ranging apparatus," Patent US 2006044546, March 2, 2006.

[17] A. C. Lewin, D. A. Orchard, et al., "A 3D camera for improved facial recognition," SPIE (Proceeding on Optics and Photonics for Counterterrorism and Crimefighting), vol. 5616, pp. 77-84, 2004.

[18] G. McDonald, D. Orchard, and A. Lewin, "A novel, rugged and robust longitudinal range finding method for formation flying missions," presented at 3rd Symposium on Formation Flying, Missions and Technologies, April 23, 2008. 